\title{
Setting up the DSPCAD Integrative Command-Line Environment: Setup Guide for DICE Version 1.2*
}

\author{
Shuvra S. Bhattacharyya, William Plishker, Kishan Sudusinghe, \\ Nimish Sane, George Zaki \\ Department of Electrical and Computer Engineering, and \\ Institute for Advanced Computer Studies \\ University of Maryland at College Park, USA \\ \{ssb, plishker, kishans, nsane, gzaki\}@umd.edu
}

June 21, 2014

This document provides instructions on setting up DICE, the DSPCAD Integrative Command Line Environment. The setup instructions provided here complement the resources provided on the DICE Project Website [1], and the DICE overview report [2]. The overview report provides general background on DICE, and is therefore useful as a preliminary orientation to the DICE package. However, it should be noted that some features and command names have changed since the publication of the overview report; for the most up-to-date information, one should consult other documentation available from the DICE Project Website [1].

\section{Setup Instructions}

This section provides information on installing DICE. The following steps outline the installation process.

1. Download the DICE package from [1], and unpack the archived download file dice.tar.gz (this will result in a singled directory called dice). Place this dice directory in the directory location where you want it to reside. This location is referred to in the remainder of this document as your DICE installation directory. For example, if one has placed the downloaded, unarchived dice directory in /users/me/import/applications, then the DICE installation directory is:

/users/me/import/applications/dice

2. Go to the parent directory of your dice_user directory (or the directory in which you want dice_user to be created if it does not yet exist). The dice_user directory is a directory in which user-specific files related to DICE are maintained. For further background about the dice_user directory, see the DICE overview report [2].

3. Run the following commands (replacing the right hand sides of the first two assignment statements appropriately for your architecture and DICE installation directory). The options for the UXARCH setting are lin (Linux), macos (Macintosh), win (Windows with Cygwin), and sol (Solaris).

\footnotetext{
${ }^{*}$ Technical Report DSPCAD-TR-2014-01, Maryland DSPCAD Research Group, Department of Electrical and Computer Engineering and Institute for Advanced Computer Studies, University of Maryland at College Park. Originally published on May 24, 2014, revised on June 21, 2014.
} 


\section{Report Documentation Page}

Form Approved

OMB No. 0704-0188

Public reporting burden for the collection of information is estimated to average 1 hour per response, including the time for reviewing instructions, searching existing data sources, gathering and maintaining the data needed, and completing and reviewing the collection of information. Send comments regarding this burden estimate or any other aspect of this collection of information,

including suggestions for reducing this burden, to Washington Headquarters Services, Directorate for Information Operations and Reports, 1215 Jefferson Davis Highway, Suite 1204, Arlington

VA 22202-4302. Respondents should be aware that notwithstanding any other provision of law, no person shall be subject to a penalty for failing to comply with a collection of information if it

does not display a currently valid OMB control number.

1. REPORT DATE

21 JUL 2014

2. REPORT TYPE

3. DATES COVERED

00-00-2014 to 00-00-2014

4. TITLE AND SUBTITLE

Setting up the DSPCAD Integrative Command-Line Environment: Setup

Guide for DICE Version 1.2

6. AUTHOR(S)

7. PERFORMING ORGANIZATION NAME(S) AND ADDRESS(ES)

University of Maryland at College Park,Department of Electrical and

Computer Engineering,College Park,MD,20742

9. SPONSORING/MONITORING AGENCY NAME(S) AND ADDRESS(ES)

5a. CONTRACT NUMBER

5b. GRANT NUMBER

5c. PROGRAM ELEMENT NUMBER

5d. PROJECT NUMBER

5e. TASK NUMBER

5f. WORK UNIT NUMBER

8. PERFORMING ORGANIZATION

REPORT NUMBER

10. SPONSOR/MONITOR'S ACRONYM(S)

11. SPONSOR/MONITOR'S REPORT

$\operatorname{NUMBER}(\mathrm{S})$

12. DISTRIBUTION/AVAILABILITY STATEMENT

Approved for public release; distribution unlimited

13. SUPPLEMENTARY NOTES

14. ABSTRACT

15. SUBJECT TERMS

16. SECURITY CLASSIFICATION OF:

a. REPORT

unclassified b. ABSTRACT

unclassified c. THIS PAGE

unclassified
17. LIMITATION OF ABSTRACT

Same as

Report (SAR)
18. NUMBER 19a. NAME OF

OF PAGES RESPONSIBLE PERSON

4 
UXARCH=macos

UXDICE=/users/me/import/applications/dice

"\$UXDICE"/setup/runme

4. If you want to run the three commands above through a script, you should place the following TWO lines at the top of the script:

\#!/usr/bin/env bash

set $-\mathrm{a}$

\section{Startup Instructions}

To use DICE in a given Bash session, one must first start up DICE within that session. Starting up involves loading necessary environment settings so that you can use all of the features in DICE.

To start up DICE, follow these steps:

1. Start a Bash shell.

2. cd to your DICE user directory (e.g., run cd /dice_user).

3. Run

source startup/dice_startup

IMPORTANT: The dice_startup file must be invoked from the dice_user directory - e.g., as opposed to running it as

source dicemin_startup

from /dice_user/startup or running it from your home directory.

As a basic test of the startup process one can run the dxversion command, which takes no arguments, from the enclosing Bash session after DICE has been started up. If DICE has been properly set up and started up, the dxversion command should execute and produce a brief message on standard output that gives the version number and other basic background information for the corresponding installation of DICE.

\section{Startup Script}

The Bash script shown in Listing 1 provides a way to start up (or "load") DICE conveniently just after starting a new Bash session. The comments in Listing 1 provide more details on how to use the script. 


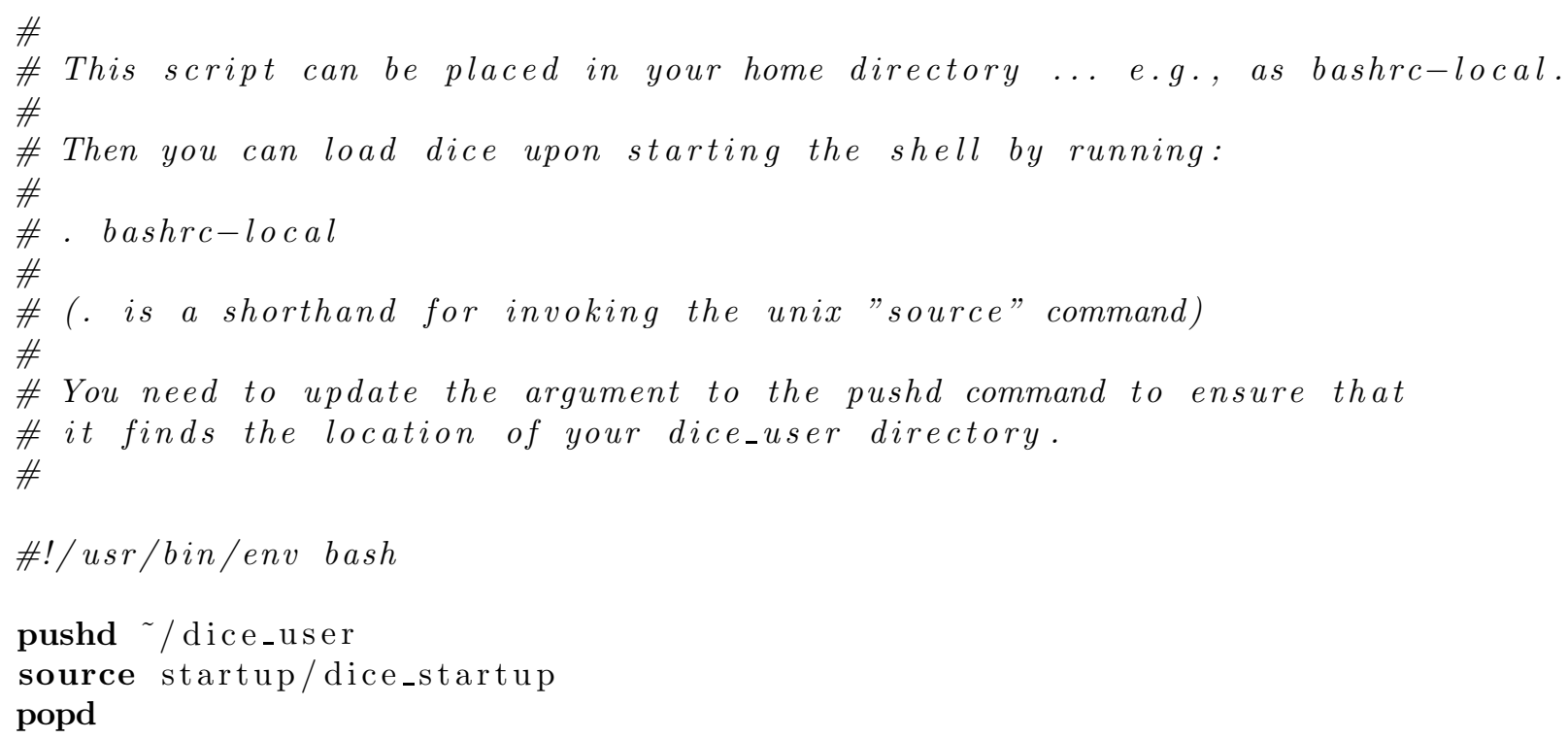

Listing 1: A Bash script for starting up DICE

\section{Updating DICE}

To update your installation of DICE to a new version (without resetting your dice-user directory), use the following steps.

1. Start up DICE (i.e., your currently-installed version of DICE).

2. Go (cd) to the directory that contains the new version of DICE.

3. Make sure that the downloaded version has been unarchived (extracted) — i.e., it should appear as the subdirectory dice (not as the file dice.tar or dice.tar.gz).

4. Run the following command:

dxupdate

5. This will install the new version of DICE, and save your previous version of DICE will in "\$UXTMP" / dice-bak.tar.gz.

6. Exit all open DICE sessions and their enclosing Bash sessions.

7. Build the new version of DICE unless instructions with the new release have been provided indicating that a build is not needed for this update. If in doubt, build the new version of DICE. To build the new version of DICE, run the following command:

dxbuild

After working through these steps successfully, one can use the new version of DICE by starting up DICE in the usual way. For example, one can start by running the dxversion command (see Section 2) to check the version number or revision date of the installed version. 


\section{Acknowledgments}

This work has been sponsored in part by the U. S. National Science Foundation, the Laboratory for Telecommunication Science, and the US Air Force Research Laboratory.

We are grateful also to the following people who have made valuable contributions to DICE, and earlier software components that have evolved into parts of DICE and dicelang: Bishnupriya Bhattacharya, Nitin Chandrachoodan, Chung-Ching Shen, Soujanya Kedilaya, and Robert Ricketts.

\section{References}

[1] "DICE project website," http://www.ece.umd.edu/DSPCAD/projects/dice/dice.htm.

[2] S. S. Bhattacharyya, W. Plishker, C. Shen, N. Sane, and G. Zaki, "The DSPCAD integrative command line environment: Introduction to DICE version 1.1," Tech. Rep. UMIACS-TR2011-10, Institute for Advanced Computer Studies, University of Maryland at College Park, 2011, http://drum.lib.umd.edu/handle/1903/11422. 